\title{
Infrared-active vibron bands associated with rare gas atom dopants isolated in solid parahydrogen
}

\author{
P.L. Raston and D.T. Anderson \\ Department of Chemistry, University of Wyoming, Laramie, WY 82071-3838, USA \\ E-mail: danderso@uwyo.edu \\ Received February 7, 2007
}

\begin{abstract}
We report high-resolution infrared absorption spectroscopic studies of the dopant-induced $Q_{1}(0)$ vibron band in solid parahydrogen crystals doped with low concentrations of rare gas atoms. The frequency, lineshape, and integrated absorption coefficient for the rare gas atom-induced $Q_{1}(0)$ vibron band are measured for $\mathrm{Ne}, \mathrm{Ar}, \mathrm{Kr}$, and $\mathrm{Xe}$. The observed lineshapes and peak maxima frequencies are sensitive to the $\mathrm{H}_{2}$ vibrational dependence of the dopant- $\mathrm{H}_{2}$ isotropic intermolecular potential. Trends observed for $\mathrm{Ar}, \mathrm{Kr}$ and $\mathrm{Xe}$ indicate the vibrational dependence is strong enough for Xe to trap the infrared-active vibron in its first solvation shell while for Ar the vibron remains delocalized. The Ne-induced feature displays a qualitatively different lineshape which is attributed to the weak intramolecular vibrational dependence of the $\mathrm{Ne}-\mathrm{H}_{2}$ intermolecular potential relative to the $\mathrm{H}_{2}-\mathrm{H}_{2}$ interaction. The lineshapes of the $\mathrm{Ar}, \mathrm{Kr}$, and Xe dopant-induced $Q_{1}(0)$ pure vibrational features agree well with recent first principles calculations.
\end{abstract}

PACS: 33.20. Ea Infrared spectra;

63.50. $+\mathbf{x}$ Vibrational states in disordered systems;

67.80.-s Solid helium and related quantum crystals.

Keywords: IR spectroscopic studies, fundamental vibrational transitions, vibron bands.

The distinguishing feature of quantum crystals, such as solid helium and solid molecular hydrogen, is the large amplitude zero-point motion of the constituent atoms or molecules about their equilibrium positions in the crystal lattice [1-5]. In solid parahydrogen $\left(p-\mathrm{H}_{2}\right)$ the rootmean-square deviation of the $p-\mathrm{H}_{2}$ molecule from its lattice site is approximately $18 \%$ of the nearest-neighbor spacing [3]. The presence of this zero-point energy dynamically inflates the molar volume of the crystal and thus solid $p-\mathrm{H}_{2}$ has a molar volume $\left(23.15 \mathrm{~cm}^{3} \cdot \mathrm{mol}^{-1}\right)$ comparable to solid argon $\left(22.42 \mathrm{~cm}^{3} \cdot \mathrm{mol}^{-1}\right)$ and significantly greater than solid neon $\left(13.31 \mathrm{~cm}^{3} \cdot \mathrm{mol}^{-1}\right)[4,6]$. This molecular motion in solid $p-\mathrm{H}_{2}$ influences the rates of intrinsically quantum relaxation processes such as ortho-para conversion and quantum diffusion whose rates are amplified by intimate short-range interactions $[4,7]$. While these quantum mechanical zero-point effects are more pronounced in low density solid helium compared to solid $p-\mathrm{H}_{2}$, because hydrogen molecules have vibrational and rotational degrees of freedom, quantum crystals of solid $p-\mathrm{H}_{2}$ have excitons (vibrons and rotons) that have no analogy in solid helium and these excitons can be used to spectroscopically probe the zero-point motion of the quantum solid [8].

Solid mixtures of $p-\mathrm{H}_{2}$ doped with low concentrations of rare gas $(\mathrm{Rg})$ atoms are of interest to investigate how the heavy dopant species perturbs the zero-point motion of the quantum crystal [4]. X-ray diffraction studies of solid $p-\mathrm{H}_{2}$ doped with $\mathrm{Ne}$, Ar, or $\mathrm{Kr}$ indicate that the molar volume of the doped solid increases and the $c / a$ ratio of the hexagonal close-packed (hcp) lattice decreases for $\mathrm{Ne}$ and $\mathrm{Ar}$ and increases for $\mathrm{Kr}$ [6,9-11]. The increase in molar volume for the $\mathrm{Ne}$ and $\mathrm{Ar}$ doped solids is in violation of Vegard's law which holds that a linear relation exists between the crystal lattice constant of an alloy and the concentration of the constituent elements [12]. Since both $\mathrm{Ne}$ and Ar have smaller molar volumes than solid $p-\mathrm{H}_{2}$, one would expect the molar volume to decrease upon adding the $\mathrm{Rg}$ atom to solid $p-\mathrm{H}_{2}$ instead of increase. This paradoxical behavior for $\mathrm{Ne}$ and Ar doped $p-\mathrm{H}_{2}$ solids led the researchers studying this effect to speculate that due to the greater mass of $\mathrm{Ne}$ the first solvation shell around the impurity is drawn inward toward the $\mathrm{Ne}$ atom as expected for a lower zero-point energy. The contraction of the first solvation shell around the Rg atom impurity 
weakens its interaction with the second solvation shell such that the second solvation shell expands causing the net increase in molar volume that is observed [6,9]. This interpretation raises basic questions about the nature of the solvent environment around the $\mathrm{Rg}$ atom in a quantum solid. The infrared spectroscopic studies presented here provide additional albeit indirect information on the «quantum solvation» environment of $\mathrm{Rg}$ atoms in solid $p-\mathrm{H}_{2}$ via detailed lineshape measurements of the solid $p-\mathrm{H}_{2}$ fundamental vibrational transition.

Although infrared (IR) transitions of isolated $\mathrm{H}_{2}$ are electric dipole forbidden, the solid possesses a rather strong infrared absorption spectrum induced via weak intermolecular interactions [13] that was first measured in neat $p-\mathrm{H}_{2}$ solids by Soots et al. [14]. The IR spectrum of solid hydrogen is therefore purely an induced spectrum arising from the electric dipole moments caused by the intermolecular forces between interacting molecules, which obviously depends on the relative separation of the $\mathrm{H}_{2}$ molecules within the solid. The infrared transition moments in solid hydrogen are induced mainly by long-range electrostatic multipole interactions among the molecules $[13,15,16]$. Doping solid $p-\mathrm{H}_{2}$ with low concentrations of closed-shell, neutral spherical dopants which rigorously do not have electric multipole moments, such as rare gas atoms, allows a qualitatively different IR induction mechanism that originates from short-range overlap interactions to be studied [17-19]. In contrast to multipole induction, overlap induction is inherently short-ranged and thus the intensity and lineshape of these $\mathrm{Rg}$ atom-induced transitions are sensitive mainly to the immediate solvation environment around the Rg impurity.

In this paper, we consider the frequency, lineshape and integrated absorption coefficients of the zero phonon $Q_{1}(0)$ transition at $\sim 4150 \mathrm{~cm}^{-1}$ induced by the presence of $\mathrm{Rg}$ atoms, corresponding to the $\mathrm{H}_{2}$ pure vibrational transition $(v=1 \leftarrow 0, J=0 \leftarrow 0)$. This transition has encoded in its high-resolution IR absorption lineshape information about the configuration distribution function of the $\mathrm{Rg}$ atom and the surrounding $p-\mathrm{H}_{2}$ solvation shell(s). The $\mathrm{Rg}$ atom doped solid $p-\mathrm{H}_{2}$ system is also amenable to first principles calculations and the present experimental results will be compared to recent theoretical studies by Hinde [19].

An important consideration in the study of the $Q_{1}(0)$ transition is the nature of the excited state. In the absence of any vibrational coupling between molecules, the $Q_{1}(0)$ transition would produce a single $v=1, J=0 p-\mathrm{H}_{2}$ molecule with an energy of $4152.2 \mathrm{~cm}^{-1}$. This energy differs from the gas-phase value because the molecule is solvated in the $p-\mathrm{H}_{2}$ crystal [15]. However, the weak intermolecular forces between two adjacent $p-\mathrm{H}_{2}$ molecules depend on the intramolecular stretching coordinate of the two molecules and this dependence leads to a mechanism for vibrational coupling [20]. This vibrational coupling delocalizes the $v=1$ vibrational state such that the $v=1$ vibrational excitation can «hop» from $p-\mathrm{H}_{2}$ molecule to $p-\mathrm{H}_{2}$ molecule within the crystal. The $v=1 p-\mathrm{H}_{2}$ vibrational state is broadened into a vibrational exciton band which extends over approximately $4 \mathrm{~cm}^{-1}$ and the exciton is termed a vibron [13].

There have been two previous experimental reports of the $\mathrm{Rg}$ atom-induced $Q_{1}(0)$ transition in solid $p-\mathrm{H}_{2}$. The first was conducted by Fajardo and Tam who developed the «rapid vapor deposition» technique utilized in this study to grow chemically doped $p-\mathrm{H}_{2}$ crystals [21]. The paper by Fajardo and Tam describes the rapid vapor deposition technique and also presents the Xe atom-induced $Q_{1}(0)$ absorption spectrum. In a later publication, Hinde et al. present data in the $Q_{1}(0)$ region for $\mathrm{N}_{2}$ and Ar dopant species [18]. Recently, Hinde has published a theoretical paper in which a model is developed for first principles calculation of the $\mathrm{Rg}$ atom-induced $Q_{1}(0)$ feature [19]. These experiments $[18,21]$ and calculations [19] are the main motivation for the present work, along with the $\mathrm{x}$-ray diffraction studies of the structural changes produced by doping solid $p-\mathrm{H}_{2}$ with $\mathrm{Rg}$ atoms [6,9-11].

\section{Experimental}

A detailed description of the experimental apparatus and sample preparation technique has been reported elsewhere [22]. $\mathrm{Rg}$ atom doped solid $p-\mathrm{H}_{2}$ crystals are prepared using rapid vapor deposition [21,23] of separate gas streams of precooled $p-\mathrm{H}_{2}$ and room temperature $\mathrm{Rg}$ onto a 1 inch diameter $\mathrm{BaF}_{2}$ substrate $(T \approx 2.5 \mathrm{~K})$ within a liquid helium bath cryostat (Janis SSVT-100). High purity $p-\mathrm{H}_{2}$ gas (99.99\%) is prepared by passing normal $\mathrm{H}_{2}$ $\left(n-\mathrm{H}_{2}\right)$ gas through a low temperature catalytic converter containing granular $\mathrm{Fe}(\mathrm{OH})_{3}$ (hydrous ferric oxide) just prior to deposition. For most of these experiments the catalytic converter was maintained at $14 \mathrm{~K}$ during deposition, which is the lowest temperature possible whilst maintaining a significant gas flow through the converter. Based on the integrated absorption of the $o-\mathrm{H}_{2}$ impurity-induced $Q_{1}(0)$ transition, we estimate the $o-\mathrm{H}_{2}$ concentration to be $\sim 100$ parts per million (ppm) in the samples discussed in this paper [23]. Both $\mathrm{Rg}$ and $p-\mathrm{H}_{2}$ gas streams impinge on the $\mathrm{BaF}_{2}$ substrate orthogonal to one another and at an angle of $45^{\circ}$ with respect to the substrate. Thermal isolation vacuum is maintained during deposition with the aid of a turbomolecular pump mounted directly to the cryostat delivering a vacuum of less than $10^{-4}$ Torr for the duration of each deposition. Sample thickness is determined to within $\pm 5 \%$ from the integrated intensities of the $Q_{1}(0)+S_{0}(0)$ or $S_{1}(0)+S_{0}(0)$ double transitions [24]. 
In these studies the $n-\mathrm{H}_{2}$ flow rate was between 170 to $300 \mathrm{mmol} \cdot \mathrm{h}^{-1}$, allowing samples from $1.6 \mathrm{~mm}$ to $2.8 \mathrm{~mm}$ thick to be grown in under an hour. The Rg atom concentration in these studies ranged from 140 to $1300 \mathrm{ppm}$, however, most samples have approximately $1000 \mathrm{ppm} \mathrm{Rg}$ atom concentrations since the induced features are relatively weak. The $\mathrm{Rg}$ concentrations cited here are ratios of the quantities of $\mathrm{Rg}$ and $\mathrm{H}_{2}$ entering the sample chamber. These values may differ from the actual Rg concentrations in the solid (due to varying sticking efficiencies of $p-\mathrm{H}_{2}$ and $\mathrm{Rg}$ atoms, among other reasons) but are estimated to be within $\pm 40 \%$ of the in situ concentrations [18]. The Rg atoms investigated were Ne (Airgas, purity: 99.999\%), Ar (Airgas, purity: 99.999\%), Kr (Scott Specialty Gases, Inc., purity: 99.995\%), and Xe (Scott Specialty Gases, Inc., purity: 99.995\%), all of which were used as received. As-deposited spectra are recorded immediately after deposition at approximately $2 \mathrm{~K}$ and are known to contain face-centered-cubic (fcc) and hexagonal close-packed (hcp) crystal domains [25]. Annealing the sample involves raising the temperature to $4.3 \mathrm{~K}$ for $\sim 40$ minutes, and should to a certain extent convert the fcc domains to the lower energy hcp crystal structures [25].

The IR absorption spectra of $\mathrm{Rg}$ atom doped solid $p-\mathrm{H}_{2}$ are recorded at resolutions ranging from $0.008 \mathrm{~cm}^{-1}$ to $0.02 \mathrm{~cm}^{-1}$ (nominal with boxcar apodization). The FTIR spectrometer (Bruker IFS 120HR) was equipped with a Tungsten source, either a $\mathrm{KBr}$ or $\mathrm{CaF}_{2}$ beam splitter, and a liquid nitrogen cooled InSb detector $\left(1850-9000 \mathrm{~cm}^{-1}\right)$. The optical path outside the spectrometer and cryostat was purged with dry $\mathrm{N}_{2}$ gas to reduce atmospheric absorptions.

\section{Results and analysis}

The $o-\mathrm{H}_{2}$ impurity-induced $Q_{1}(0)$ feature has been extensively studied both experimentally [26-29] and theoretically $[13,15,16]$. The dipole moment induced in $p-\mathrm{H}_{2}$ by an $o-\mathrm{H}_{2}$ molecule via intermolecular forces consists of two main parts; the overlap dipole moment and the electric quadrupole-induced dipole moment due to the rotationally averaged quadrupole moment of $o-\mathrm{H}_{2}$. For $o-\mathrm{H}_{2}-p-\mathrm{H}_{2}$ pairs at the nominal $3.79 \AA$ nearest-neighbor spacing in the crystal [3], the overlap dipole moment is small compared to the quadrupole-induced moment and therefore assumed not to contribute to the $o-\mathrm{H}_{2}$ induced $Q_{1}(0)$ transition. An important distinction between the two induction mechanisms is that overlap induction is isotropic while quadrupole induction is anisotropic. In addition, overlap induction falls off exponentially with respect to distance, while quadrupole induction decays rather slowly with increasing distance $\left(1 / R^{4}\right)$ and thus activates vibrons with substantial amplitude on non-nearest neighbor $p-\mathrm{H}_{2}$ molecules [19]. The quadrupole induction mechanism, IR selection rules, and the fact that the upper

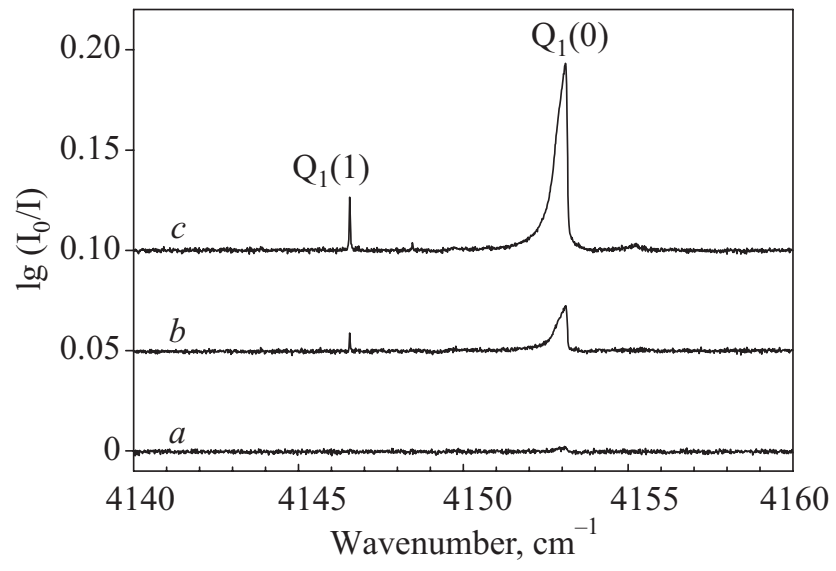

Fig. 1. Infrared absorption spectra in the $4140-4160 \mathrm{~cm}^{-1}$ region recorded at $1.9 \mathrm{~K}$ for as-deposited neat $p-\mathrm{H}_{2}$ crystals with $o-\mathrm{H}_{2}$ concentrations as follows: $100 \mathrm{ppm}(a), 1000 \mathrm{ppm}(b)$, $5200 \mathrm{ppm}(c)$. The $o-\mathrm{H}_{2} Q_{1}(1)$ and $o-\mathrm{H}_{2}$ impurity-induced $Q_{1}(0)$ absorption features are labeled accordingly.

state is a delocalized vibron results in the distinct broad asymmetric lineshape observed for the $o-\mathrm{H}_{2}$ impurity-induced $Q_{1}(0)$ transition where the IR absorption line profile is similar to the calculated density of vibron Bloch states [30].

The $o-\mathrm{H}_{2}$ induced $Q_{1}(0)$ transition is shown in Fig. 1 for a series of $p-\mathrm{H}_{2}$ solids with low concentrations of $o-\mathrm{H}_{2}$. The spectra shown in Fig. 1 are all as-deposited spectra recorded at $1.9 \mathrm{~K}$ with $o-\mathrm{H}_{2}$ concentrations as follows: trace $(a) \sim 100 \mathrm{ppm}$, trace $(b) 1000 \mathrm{ppm}$, and trace (c) $5200 \mathrm{ppm}$. The $Q_{1}(0)$ feature at $4153.1 \mathrm{~cm}^{-1}$ exhibits the broad asymmetric lineshape which maps out the subset of delocalized $Q_{1}(0)$ vibrons whose spatial wave functions achieve nonzero overlap with the quadrupolar electrostatic field of the $o-\mathrm{H}_{2}$ dopant. In contrast, the $Q_{1}(1)$ transition $(v=1 \leftarrow 0, J=0 \leftarrow 0)$ of the $o-\mathrm{H}_{2}$ dopant itself is observed at $4146.55 \mathrm{~cm}^{-1}$ with a narrow linewidth, indicating the vibrational excitation is extensively localized on $o-\mathrm{H}_{2}$ [31]. The large vibration-rotation interaction in $\mathrm{H}_{2}$ shifts the $Q_{1}(1)$ transition $\sim 6.6 \mathrm{~cm}^{-1}$ to lower energy than the $Q_{1}(0)$ transition, thus the $o-\mathrm{H}_{2}$ vibrational excited state is below the $p-\mathrm{H}_{2}$ vibron band which substantially localizes the vibrational excitation on the $o-\mathrm{H}_{2}$ dopant.

To minimize the intensity of the $o-\mathrm{H}_{2} Q_{1}(1)$ and $o-\mathrm{H}_{2}$ impurity-induced $Q_{1}(0)$ absorption features in the present study, the crystals were grown with the lowest concentration of $o-\mathrm{H}_{2}$ that could be achieved (approximately $100 \mathrm{ppm}$ ), a representative spectrum at this $o-\mathrm{H}_{2}$ concentration is shown in trace $(a)$ of Fig. 1. Since the ortho to para conversion rate in the solid at these low $o-\mathrm{H}_{2}$ concentrations is on the order of days, all the spectra reported here in the $Q_{1}(0)$ region contain a small feature due to the $o$ - $\mathrm{H}_{2}$ impurity-induced $Q_{1}(0)$ absorption [3,32]. The frequency and lineshape of this $Q_{1}(0)$ absorption feature are 


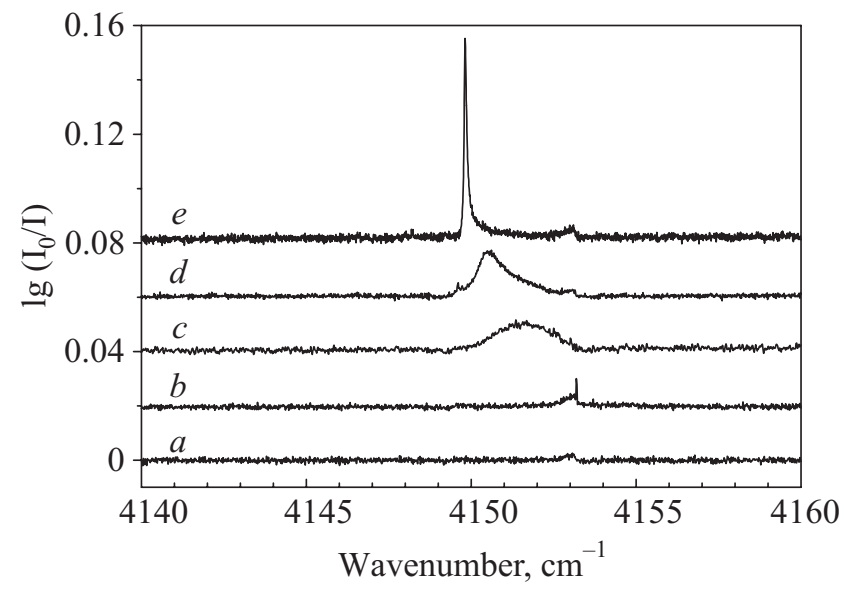

Fig. 2. Infrared absorption spectra in the $4140-4160 \mathrm{~cm}^{-1}$ region displaying the $\mathrm{Rg}$ atom-induced $Q_{1}(0)$ absorption features. All spectra are for as-deposited samples recorded at $2 \mathrm{~K}$. Trace $(a)$ is for a $2.8(1) \mathrm{mm}$ thick neat $p-\mathrm{H}_{2}$ solid containing $\sim 100 \mathrm{ppm}$ of $o-\mathrm{H}_{2}$. The other spectra are $\mathrm{Rg}$ atom doped samples with thicknesses and $\mathrm{Rg}$ atom concentrations as follows: 2.8(1) mm, $1000 \mathrm{ppm} \mathrm{Ne}(b), 1.8(1) \mathrm{mm}, 1300 \mathrm{ppm}$ $\operatorname{Ar}(c), 1.6(1) \mathrm{mm}, 790 \mathrm{ppm} \mathrm{Kr}(d)$, and 2.5(1) mm, $260 \mathrm{ppm}$ $\mathrm{Xe}(e)$.

well-known and at these low $o-\mathrm{H}_{2}$ concentrations this peak does not significantly mask the $\mathrm{Rg}$ atom-induced $Q_{1}(0)$ feature.

A series of spectra in the $Q_{1}(0)$ region are shown in Fig. 2 for $p-\mathrm{H}_{2}$ crystals doped with different $\mathrm{Rg}$ atoms. The spectrum shown in trace $(a)$ of Fig. 2 is for a 2.8(1) mm thick neat $p$ - $\mathrm{H}_{2}$ crystal, no $\mathrm{Rg}$ atom dopant, and illustrates the small $o-\mathrm{H}_{2}$ impurity-induced $Q_{1}(0)$ absorption feature that is present in all the spectra due to residual $o-\mathrm{H}_{2}$. Traces $(b)-(e)$ are recorded at $2 \mathrm{~K}$ for as-deposited $p-\mathrm{H}_{2}$ samples containing $\mathrm{Ne}, \mathrm{Ar}, \mathrm{Kr}$, and $\mathrm{Xe}$, respectively. Note that attempts to observe the $\mathrm{He}$ atom-induced $Q_{1}(0)$ absorption were unsuccessful, even for samples deposited with relatively high $\mathrm{He}$ gas flow rates. Possible reasons for the lack of a He induced $Q_{1}(0)$ feature could be the inability of the $p-\mathrm{H}_{2}$ crystals to trap sufficient concentrations of $\mathrm{He}$ atoms and/or that the $\mathrm{He}$ induced $Q_{1}(0)$ feature is extremely weak and below current detection limits.

Examination of Fig. 2 illustrates the trend that the Rg atom-induced $Q_{1}(0)$ absorption feature shifts to lower energy and narrows going from $\mathrm{Ar}$ to $\mathrm{Kr}$ to $\mathrm{Xe}$. Note that the Ne induced $Q_{1}(0)$ feature does not follow this trend, with almost no induced signal except for a sharp absorption feature right at the blue edge of the $o-\mathrm{H}_{2}$ impurity-induced $Q_{1}(0)$ feature. The spectra shown in Fig. 2 were recorded for $\mathrm{Rg}$ atom concentrations ranging from 260 to $1300 \mathrm{ppm}$. To make comparison with solid $p-\mathrm{H}_{2}$ literature values $[27,33,34]$, integrated absorption coefficients $(\widetilde{\alpha})$ for the experimental decadic absorption spectra recorded as a function of wavenumber are determined using the equation,

$$
\widetilde{\alpha}=\frac{2.303 c}{N_{R g} d<\widetilde{v}>} \int_{\text {band }} \lg \left[\frac{I_{0}}{I}\right] d \widetilde{v}
$$

in which $c$ is the speed of light, $N_{R g}$ is the number density of $\mathrm{Rg}$ atoms (atoms $\mathrm{cm}^{-3}$ ), $d$ is the optical path length of the sample, and $\langle\widetilde{v}\rangle$ is the centroid of the absorption band. The number density of $\mathrm{Rg}$ atoms is calculated using the fractional dopant concentration and the number density [4] of solid $p-\mathrm{H}_{2}$ at $1 \mathrm{He}$ temperatures $\left(N_{p-\mathrm{H}_{2}}=\right.$ $=2.601 \cdot 10^{22}$ molecules $\left.\cdot \mathrm{cm}^{-3}\right)$. The frequencies of the peak maxima, the full width at half maximum (FWHM), and the integrated absorption coefficients for these $\mathrm{Rg}$ atom-induced $Q_{1}(0)$ features are given in Table 1.

Table 1. Measured Rg atom-induced $Q_{1}(0)$ transition frequency, FWHM, centroid frequency, and integrated absorption coefficient

\begin{tabular}{c|c|c|c|c}
\hline \hline $\mathrm{Rg}$ & $\begin{array}{c}{ }^{\max } \\
\mathrm{cm}^{-1}\end{array}$ & $\begin{array}{c}\text { FWHM, } \\
\mathrm{cm}^{-1}\end{array}$ & $\begin{array}{c}<\widetilde{v}> \\
\mathrm{cm}^{-1}\end{array}$ & $\begin{array}{c}\tilde{\alpha}, \\
\mathrm{cm}^{3} \cdot \mathrm{s}^{-1 *}\end{array}$ \\
\hline \hline $\mathrm{Ne}$ & $4153.20(1)$ & $0.03(1)$ & $4153.20(1)$ & $1(2) \cdot 10^{-15}$ \\
$\mathrm{Ar}$ & $4151.6(1)$ & $2.0(1)$ & $4151.6(1)$ & $5.1(4) \cdot 10^{-14}$ \\
$\mathrm{Kr}$ & $4150.5(1)$ & $1.1(1)$ & $4150.8(1)$ & $1.0(1) \cdot 10^{-13}$ \\
$\mathrm{Xe}$ & $4149.82(1)$ & $0.11(1)$ & $4149.9(1)$ & $1.7(2) \cdot 10^{-13}$ \\
\hline \hline
\end{tabular}

Notes: * reported uncertainties represent estimated statistical errors only

The Rg atom-induced $Q_{1}(0)$ spectra show no discernable temperature dependence over the $1.9 \mathrm{~K}$ to $4.5 \mathrm{~K}$ temperature range studied. In addition, annealing the as-deposited samples at $4.3 \mathrm{~K}$ for $\sim 1$ hour had little if any effect on the $\mathrm{Rg}$ atom-induced $Q_{1}(0)$ absorption features. Figure 3 shows the effects of annealing a $440 \mathrm{ppm} \mathrm{Kr}$ doped $p-\mathrm{H}_{2}$ solid. Sharp features at 4149.02, 4149.61, and $4149.72 \mathrm{~cm}^{-1}$ grow in upon annealing, indicating they may be due to the presence of $\mathrm{Kr}$ atom dimers or clusters. The main conclusion, however, is the $\mathrm{Rg}$ atom-induced $Q_{1}(0)$ absorption lineshape is not temperature dependent and shows little change upon annealing.

\section{Discussion}

The lineshape of the $\mathrm{Rg}$ atom-induced $Q_{1}(0)$ feature can be modeled with a tight-binding-like Hamiltonian in which nearest-neighbor $p-\mathrm{H}_{2}$ molecules are coupled by off diagonal matrix elements that represent the «hopping» of the vibrational excitation from one molecule to the next $[19,35]$. The Hamiltonian can be written as

$$
\hat{H}=\sum_{k} E_{k}|k\rangle\left\langle k\left|+\lambda \sum_{k, n}\right| k\right\rangle\langle n|,
$$


where $E_{k}$ is the $Q_{1}(0)$ transition energy for vibrational excitation localized on molecule $k$ and $\lambda$ represents the vibrational coupling between nearest-neighbor molecules. Intermolecular interactions in the solid [16] reduce $E_{k}$ from its gas-phase value of $E_{k}=4161.1 \mathrm{~cm}^{-1}$ to $E_{k}=$ $=4152.2 \mathrm{~cm}^{-1}$. The vibron hopping parameter $\lambda$ has been determined from experiment $[29,36]$ and theory $[15,30]$ to be $\lambda=-0.25 \mathrm{~cm}^{-1}$. The sign of the vibron coupling parameter indicates the out-of-phase (antisymmetric) combination of vibrational states is higher in energy than the in-phase (symmetric) combination. Since the equilibrium intermolecular distance [4] in the crystal (3.783 $\AA$ ) is much larger than the equilibrium distance [37] of the intermolecular pair potential (3.41 $\AA$ ), molecules sense mostly the attractive part of the intermolecular potential due to the $1 / R^{6}$ dispersion term and thus the vibrational coupling depends primarily on the intramolecular stretching dependence of the $C_{6}$ dispersion coefficient [38].

In the $\mathrm{Rg}$ atom doped $p-\mathrm{H}_{2}$ crystal, the $Q_{1}(0)$ transition energy $E_{k}$ for $p-\mathrm{H}_{2}$ molecules that are nearest-neighbors of the $\mathrm{Rg}$ atom will differ from $p-\mathrm{H}_{2}$ molecules distant from the $\mathrm{Rg}$ atom. In model studies conducted by Hinde [19], this perturbation of $E_{k}$ caused by the presence of the $\mathrm{Rg}$ atom dopants was taken into account by introducing a parameter $\Delta E$ that quantifies the shift in the $Q_{1}(0)$ transition energy for $p-\mathrm{H}_{2}$ molecules that are nearest-neighbors to the $\mathrm{Rg}$ atom. In the calculations, $E_{k}=4152.2 \mathrm{~cm}^{-1}-\Delta E$ for nearest-neighbors, while $E_{k}$ remains $4152.2 \mathrm{~cm}^{-1}$ for all other $p-\mathrm{H}_{2}$ molecules. The doped $p-\mathrm{H}_{2}$ crystal is treated as a random close-packed solid consisting of a randomly chosen sequence of close-packed planes of $p-\mathrm{H}_{2}$ molecules. Hinde then studied how the line profile of the dopant-induced $Q_{1}(0)$ vibron band depends on the detuning parameter $\Delta E$. At low values of $\Delta E$, the dopant-induced $Q_{1}(0)$ absorption feature shows substantial IR intensity over the entire vibron band ranging from 4149.5 to $4153.1 \mathrm{~cm}^{-1}$. As $\Delta E$ increases, the dopant-induced absorption feature shifts to the red and sharpens (see Fig. 2 in Ref. 19).

The $\Delta E$ dependence of the lineshape predicted by the calculations of Hinde reproduces the general trend observed experimentally for $\mathrm{Ar}, \mathrm{Kr}$, and $\mathrm{Xe}$. Based upon measurements of the vibrational shifts of the $Q_{1}(0)$ transition in the isolated gas-phase van der Waals dimers $\left(\mathrm{Rg}-p-\mathrm{H}_{2}\right)[39,40]$, one can roughly estimate the value of the $\Delta E$ parameter in the $p-\mathrm{H}_{2}$ solid. The vibrational shifts, $\Delta v=v_{\mathrm{gas}}-v_{v d w}$, for the $\mathrm{Rg}-p-\mathrm{H}_{2}$ van der Waals dimers are reproduced in Table 2 and can be used as estimates of the $\Delta E$ values; that is, $\Delta E$ equals $1.09,1.63$, and $2.51 \mathrm{~cm}^{-1}$ for $\mathrm{Ar}, \mathrm{Kr}$, and $\mathrm{Xe}$, respectively. Comparison of Fig. 2 in Ref. 19 with the experimental spectra in Fig. 2 illustrates that the calculated lineshapes for $\Delta E$ parameters equal to $1,1.5$ and $2 \mathrm{~cm}^{-1}$ nearly quantitatively reproduce the experimentally measured lineshapes and shifts for $\mathrm{Ar}, \mathrm{Kr}$,
Table 2. The vibrational shifts of the $Q_{1}(0)$ transition for the isolated $\mathrm{Rg}-p-\mathrm{H}_{2}$ van der Waals dimer (Ref. 40). The intermolecular spacing $R_{0}$ determined from analysis of the rotational $B$ constant are also reported (Ref. 40)

\begin{tabular}{c|c|c}
\hline \hline $\mathrm{Rg}$ & $\Delta v=v_{\mathrm{gas}}-v_{v d w}, \mathrm{~cm}^{-1}$ & $R_{0}, \AA$ \\
\hline \hline $\mathrm{Ne}$ & 0.02 & 3.99 \\
$\mathrm{Ar}$ & 1.09 & 3.94 \\
$\mathrm{Kr}$ & 1.63 & 4.07 \\
$\mathrm{Xe}$ & 2.51 & 4.25 \\
\hline \hline
\end{tabular}

and Xe. However, the van der Waals dimer $Q_{1}(0)$ redshifts can not be directly equated with the $\Delta E$ parameter, because the vibrationally averaged distance between the $\mathrm{Rg}$ atom and $p-\mathrm{H}_{2}$ molecule in the isolated van der Waals dimer (see Table 2) will most likely differ from the average separation in the $p-\mathrm{H}_{2}$ solid. While $\Delta E$ was used as an adjustable parameter in the published work by Hinde, currently these researchers are trying to calculate $\Delta E$ directly using path integral Monte Carlo simulations and ab initio calculations of the interaction-induced dipole moment $[41,42]$.

The evolution in the lineshape of the $\mathrm{Rg}$ atom-induced $Q_{1}(0)$ feature going from $\mathrm{Ar}$ to $\mathrm{Kr}$ to Xe reflects a transition from a delocalized IR-active vibron for $\mathrm{Ar}$ to a strongly localized IR-active vibron for Xe. As the magnitude of $\Delta E$ increases, it becomes more difficult for a vibrational excitation localized in the dopant's first solvation shell to hop to the next solvation shell and move away from the dopant. Thus, as the interaction with the $\mathrm{Rg}$ dopant shifts the vibrational frequency of the $p-\mathrm{H}_{2}$ molecules in the first solvation shell to lower energies, these $p-\mathrm{H}_{2}$ molecules become effectively decoupled from the bulk and the transition sharpens. Furthermore, the

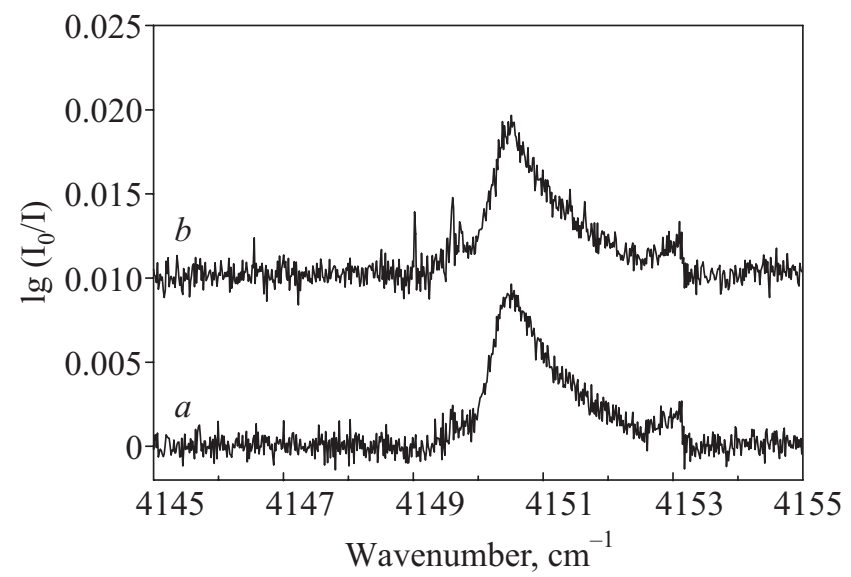

Fig. 3. Infrared absorption spectra in the $4145-4155 \mathrm{~cm}^{-1}$ region of a $1.6(1) \mathrm{mm}$ thick, $440 \mathrm{ppm} \mathrm{Kr}$ doped $p-\mathrm{H}_{2}$ solid. Trace $(a)$ is the as-deposited sample recorded at $2.1 \mathrm{~K}$ and trace $(b)$ is the annealed sample recorded at $2 \mathrm{~K}$. 
short-range nature of the overlap induction mechanism only activates $p-\mathrm{H}_{2}$ molecules in the first solvation shell of the $\mathrm{Rg}$ atom. Thus, the picture that emerges for the Xe atom-induced $Q_{1}(0)$ vibron is a $v=1$ excitation delocalized on the first solvation shell of the Xe atom, but trapped around the $\mathrm{Xe}$ atom with negligible amplitude on non-nearest neighbors. In contrast, the vibron induced by the Ar atom remains delocalized, with significant vibrational amplitude on non-nearest neighbor $p-\mathrm{H}_{2}$ molecules.

A comparison of Hinde's lineshape calculations with the spectra in Fig. 2 also supports overlap induction as the operative induction mechanism. In the case of the $o-\mathrm{H}_{2}$ dopant, both quadrupole and overlap induction mechanisms are operative, however quadrupole induction tends to dominate at the relatively large nearest-neighbor spacing of the solid and therefore masks the effects of overlap induction. Quadrupole induction is anisotropic and falls off as $1 / R^{4}$, while overlap induction is isotropic and falls off as $\exp \left(-R / R_{0}\right)$. Both induction mechanisms generate net transition dipole moments through sums of pairwise induction and thus can suffer from cancellation effects. In the high symmetry hep environment of the solid, the isotropic overlap induction mechanism relies on the symmetry breaking due to the presence of the Rg atom to generate net transition dipole moments that differ from zero since the induction mechanism is isotropic. Thus, the lineshape of the $\operatorname{Rg}$ atom-induced $Q_{1}(0)$ feature is qualitatively different from the $o-\mathrm{H}_{2}$ induced $Q_{1}(0)$ feature.

As discussed in the results section, the $\mathrm{Ne}$ induced $Q_{1}(0)$ lineshape does not follow the trend measured for $\mathrm{Ar}, \mathrm{Kr}$, and $\mathrm{Xe}$. If $\mathrm{Ne}$ did follow the trend, one would expect a lineshape reminiscent of the $\operatorname{Ar}$ induced $Q_{1}(0)$ feature since the detuning parameter $\Delta E$ for $\mathrm{Ne}$ would be expected to be even smaller. While the $Q_{1}(0)$ absorption induced by $\mathrm{Ne}$ is very weak, we assign the sharp feature at the blue edge of the residual $o-\mathrm{H}_{2}$ induced $Q_{1}(0)$ feature to Ne. Unlike Ar, $\mathrm{Kr}$ and $\mathrm{Xe}$, the shallow attractive well of the $\mathrm{Ne}-\mathrm{H}_{2}$ intermolecular potential is comparable in magnitude to the $\mathrm{H}_{2}-\mathrm{H}_{2}$ intermolecular potential well. The corresponding $\mathrm{Rg}-\mathrm{H}_{2}$ isotropic pair potentials $[43,44]$ for all the rare gases are plotted in Fig. 4. The Ar, $\mathrm{Kr}$, and $\mathrm{Xe}$ potentials all have significantly deeper wells compared to the isotropic $\mathrm{H}_{2}-\mathrm{H}_{2}$ pair potential determined by Silvera and Goldman [37] with equilibrium separation $r_{m}=$ $=3.41 \AA$ and well depth $\varepsilon=23.8 \mathrm{~cm}^{-1}$. Therefore, we surmise that replacing one of the nearest-neighbor $p-\mathrm{H}_{2}$ molecules of a central $p-\mathrm{H}_{2}$ molecule with an $\mathrm{Ar}, \mathrm{Kr}$, or Xe atom results in a redshift of that $p$ - $\mathrm{H}_{2}$ molecule's vibrational frequency. However, since the $\mathrm{Ne}-\mathrm{H}_{2}$ and $\mathrm{H}_{2}-\mathrm{H}_{2}$ potentials are very similar with near identical equilibrium distances and well depths, it seems that replacing one of the nearest-neighbors of a central $p-\mathrm{H}_{2}$ molecule with $\mathrm{Ne}$ results in a net blue shift of the $\mathrm{H}_{2}$ vibrational frequency.

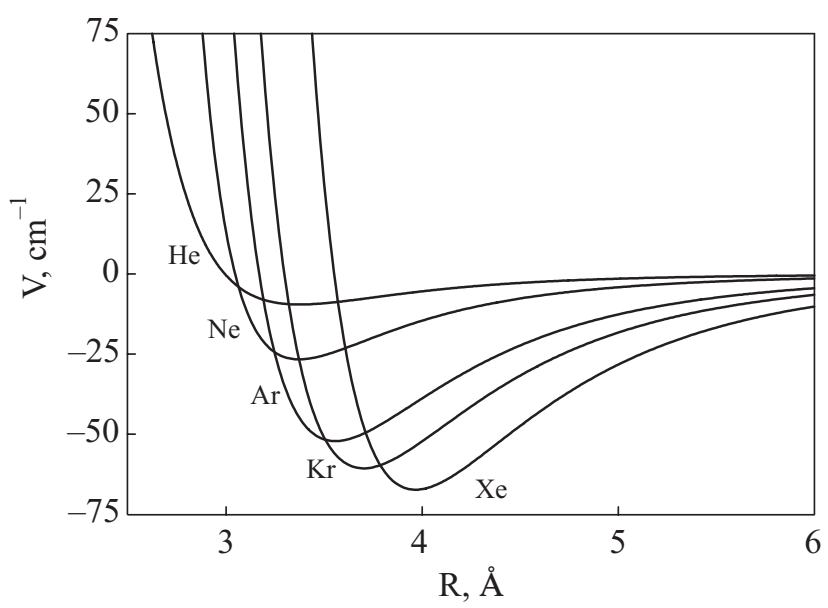

Fig. 4. Plots of the isotropic $\mathrm{Rg}-\mathrm{H}_{2}$ pair potentials for $\mathrm{He}, \mathrm{Ne}$, $\mathrm{Ar}, \mathrm{Kr}$, and $\mathrm{Xe}$. Note the $\mathrm{Ne}-\mathrm{H}_{2}$ potential has a significantly shallower well than $\mathrm{Ar}, \mathrm{Kr}$, or $\mathrm{Xe}$. The $\mathrm{Ne}, \mathrm{Ar}, \mathrm{Kr}$, and $\mathrm{Xe}$ potentials are reproduced using parameters in Ref. 44 and the He potential from Ref. 43.

We speculate based on examination of the intermolecular potentials that unlike the other $\mathrm{Rg}$ atoms, Ne shifts the transition frequency of adjacent $p-\mathrm{H}_{2}$ molecules to higher energies instead of lower relative to the pure $p-\mathrm{H}_{2}$ solid vibron band. It would therefore be interesting to model this transition by using a negative $\Delta E$ value in Eq. (2) to see if the lineshape calculations reproduce the sharp $\mathrm{Ne}$ induced $Q_{1}(0)$ absorption feature shown in Fig. 2.

The other experimental observable measured in these studies is the integrated absorption strengths of the Rg atom-induced $Q_{1}(0)$ absorption features. Overlap induction relies on the overlap of the charge clouds of the Rg atom and $p-\mathrm{H}_{2}$ molecule. Overlap induction involves both exchange and charge deformation terms [17]. Van Kranendonk has shown that the magnitude of the induced dipole moment when a vibrating-translating $\mathrm{H}_{2}$ interacts with a impurity atom may be approximated empirically by the relation $\mu_{\text {ind }}=\xi \exp (-R / \rho)$, where $\xi$ and $\rho$ are parameters that represent the strength and range, respectively, of the short-range induced moment [17]. The integrated absorption strengths depend on the separation $(R)$ between the $p-\mathrm{H}_{2}$ molecule and $\mathrm{Rg}$ atom sampled in the solid at liquid helium temperatures, and on the strength of the interaction as measured for example by the well depth of the intermolecular potential. Thus, the integrated absorption strengths are strongly correlated to both the well depth and equilibrium separation of the $\mathrm{Rg}-p-\mathrm{H}_{2}$ intermolecular potential.

The greatest $\mathrm{Rg}$ atom-induced $Q_{1}(0)$ integrated absorption strength measured in this study for Xe is more than an order of magnitude greater than the absorption strength for the $o-\mathrm{H}_{2}$ impurity-induced $Q_{1}(0)$ feature. The integrated absorption coefficient per single ortho-mole- 
cule per unit volume is measured [27] by Gush et al. to be $2.2 \cdot 10^{-14} \mathrm{~cm}^{3} \cdot \mathrm{s}^{-1}$. Thus, the Rg atom-induced $Q_{1}(0)$ features for $\mathrm{Ar}, \mathrm{Kr}$, and $\mathrm{Xe}$ all have greater absorption coefficients than the $o-\mathrm{H}_{2}$ impurity-induced $Q_{1}(0)$ feature and can be used to quantify the concentration of $\mathrm{Rg}$ atom down to approximately the $50 \mathrm{ppm}$ level using infrared absorption spectroscopy. Theoretical modeling of the induced intensities can also be used to estimate $\langle R>$, the average value of the separation of the $\mathrm{Rg}$ atom and the first solvation shell sampled in the solid, and thus by iterating between experiment and simulation one may be able to gain insight into how the heavy $\mathrm{Rg}$ atom perturbs the zero-point motion of the $p-\mathrm{H}_{2}$ crystal.

\section{Conclusions}

In this paper we have presented IR spectroscopic studies of $\mathrm{Rg}$ atom impurity-induced $Q_{1}(0)$ fundamental vibrational transitions for solid $p-\mathrm{H}_{2}$ doped with $\mathrm{Ne}, \mathrm{Ar}, \mathrm{Kr}$, and Xe impurities. The impurities induce IR activity through short-range overlap induction interactions. The general trend in lineshape measured for $\mathrm{Ar}, \mathrm{Kr}$, and $\mathrm{Xe}$ are well reproduced by the calculations of Hinde [19]. The Ar induced $Q_{1}(0)$ vibron is a loosely bound IR-active vibron with a broad lineshape while in contrast the Xe induced $Q_{1}(0)$ vibron is tightly bound to the first solvation shell, evident by the relatively sharp induced band. The lineshape of the Ne atom-induced $Q_{1}(0)$ feature does not follow the trend established for $\mathrm{Ar}, \mathrm{Kr}$ and $\mathrm{Xe}$ and we speculate that this difference is due to the subtle balance between $\mathrm{Ne}-\mathrm{H}_{2}$ and $\mathrm{H}_{2}-\mathrm{H}_{2}$ intermolecular interactions within the solid. In contrast to $\mathrm{Ar}, \mathrm{Kr}$ and $\mathrm{Xe}$ which redshift the vibron band, doping with Ne results in a blue shift of the vibron energy compared to the pure solid's vibron band. Interestingly, Hinde's model calculations do not predict an induced feature similar to the one measured for $\mathrm{Ne}$, and we believe this may be due to the fact that Hinde did not consider blue-shifted vibrons in the published work [19]. It is hoped that the spectroscopic data presented here will motivate further theoretical studies so that the information about the $\mathrm{Rg}-\mathrm{H}_{2}$ pair distribution function encoded in these spectroscopic data may be discerned and quantified. Understanding how the heavy Rg atom perturbs the large amplitude zero-point motion of a quantum solid is of general interest in many-body quantum mechanics.

This work was funded in part by a grant from the Petroleum Research Fund, administered by the American Chemical Society, an award from the Research Corporation, and by a grant from the Chemistry Division of the National Science Foundation (Grant No. CHE-0316268). The authors would like to thank Dr. M.E. Fajardo for making his IR spectral data available to us and acknowledge helpful discussions throughout the course of this work with Prof. R.J. Hinde.
1. L.H. Nosanow, Phys. Rev. 146, 120 (1966).

2. R.A. Guyer, R.C. Richardson, and L.I. Zane, Rev. Mod. Phys. 43, 532 (1971).

3. I.F. Silvera, Rev. Mod. Phys. 52, 393 (1980).

4. Physics of Cryocrystals, V.G. Manzhelii, Yu.A. Freiman, M.L. Klein, and A.A. Maradudin (eds.), AIP Press, Woodbury, New York (1997).

5. E. Polturak and N. Gov, Contemp. Phys. 44, 145 (2003).

6. N.N. Galtsov, A.I. Prokhvatilov, G.N. Shcherbakov, and M.A. Strzhemechny, Fiz. Nizk. Temp. 29, 1036 (2003) [Low Temp. Phys. 29, 784 (2003)].

7. H. Meyer, Fiz. Nizk. Temp. 24, 507 (1998) [Low Temp. Phys. 24, 381 (1998)].

8. H. Katsuki, M. Fushitani, and T. Momose, Fiz. Nizk. Temp. 29, 1093 (2003) [Low Temp. Phys. 29, 832 (2003)].

9. A.S. Baryl'nik, A.I. Prokhvatilov, M.A. Strzhemechny, and G.N. Shcherbakov, Fiz. Nizk. Temp. 19, 625 (1993) [Low Temp. Phys. 19, 447 (1993)].

10. A.S. Baryl'nik, A.I. Prokhvatilov, and G.N. Shcherbakov, Fiz. Nizk. Temp. 21, 787 (1995) [Low Temp. Phys. 21, 607 (1995)].

11. M.A. Strzhemechny, N.N. Galtsov, and A.I. Prokhvatilov, Fiz. Nizk. Temp. 29, 649 (2003) [Low Temp. Phys. 29, 522 (2003)].

12. L. Vegard, Z. Phys. 5, 17 (1921).

13. J. Van Kranendonk, Solid Hydrogen-Theory of the properties of solid $\mathrm{H}_{2}, \mathrm{HD}$, and $\mathrm{D}_{2}$, Plenum Press, New York (1983).

14. V. Soots, E.J. Allen, and H.L. Welsh, Can. J. Phys. 40, 9 (1962).

15. V.F. Sears and J. Van Kranendonk, Can. J. Phys. 42, 980 (1964).

16. J. Van Kranendonk and G. Karl, Rev. Mod. Phys. 40, 531 (1968).

17. J. Van Kranendonk, Physica 24, 347 (1958).

18. R.J. Hinde, D.T. Anderson, S. Tam, and M.E. Fajardo, Chem. Phys. Lett. 356, 355 (2002).

19. R.J. Hinde, J. Chem. Phys. 119, 6 (2003).

20. R.J. Hinde, Few-Body Syst. 38, 187 (2006).

21. M.E. Fajardo and S. Tam, J. Chem. Phys. 108, 4237 (1998).

22. K. Yoshioka and D.T. Anderson, J. Chem. Phys. 119, 4731 (2003).

23. S. Tam and M.E. Fajardo, Rev. Sci. Instrum. 70, 1926 (1999).

24. S. Tam and M.E. Fajardo, Appl. Spectrosc. 55, 1634 (2001).

25. S. Tam, M.E. Fajardo, H. Katsuki, H. Hoshina, T. Wakabayashi, and T. Momose, J. Chem. Phys. 111, 4191 (1999).

26. E.J. Allin, W.F.J. Hare, and R.E. MacDonald, Phys. Rev. 98, 554 (1955).

27. H.P. Gush, W.F.J. Hare, E.J. Allin, and H.L. Welsh, Can. J. Phys. 38, 176 (1960).

28. W. Ivancic, T.K. Balasubramanian, J.R. Gaines, and K.N. Rao, J. Chem. Phys. 74, 1508 (1981).

29. R.A. Steinhoff, K.V.S.R. Apparao, D.W. Ferguson, K.N. Rao, B.P. Winnewisser, and M. Winnewisser, Can. J. Phys. 72, 1122 (1994).

30. S.K. Bose and J.D. Poll, Can. J. Phys. 68, 159 (1990).

31. M.-C. Chan, M. Okamura, C.M. Gabys, L.-W. Xu, B.D. Rehfuss, and T. Oka, Phys. Rev. Lett. 66, 2060 (1991). 
32. F. Schmidt, Phys. Rev. B10, 4480 (1974).

33. Q. Ma, R.H. Tipping, and J.D. Poll, Phys. Rev. B39, 132 (1989).

34. A.P. Mishra, R. D'Souza, and T.K. Balasubramanian, Phys. Rev. B70, 174303 (2004).

35. J.L. Feldman, J.H. Eggert, J. De Kinder, R.J. Hemley, H. Mao, and D. Schoemaker, Phys. Rev. Lett. 74, 1379 (1995).

36. M.-C. Chan, L.-W. Xu, C.M. Ganrys, and T. Oka, J. Chem. Phys. 95, 9404 (1991)

37. I.F. Silvera and V.V. Goldman, J. Chem. Phys. 69, 4209 (1978).

38. Y. Zhang, T.J. Byers, M.-C. Chan, T. Momose, K.E. Kerr, D.P. Weliky, and T. Oka, Phys. Rev. B58, 218 (1998).
39. A.R.W. McKellar and H.L. Welsh, J. Chem. Phys. 55, 595 (1971).

40. A.R.W. McKellar and H.L. Welsh, Can. J. Phys. 50, 1458 (1972).

41. R.J. Hinde, J. Chem. Phys. 124, 154309 (2006).

42. R.J. Hinde, J. Chem. Phys. 122, 144304 (2005).

43. W.R. Rodwell and G. Scoles, J. Phys. Chem. 86, 1053 (1982).

44. R.J. Le Roy and J. Van Kranendonk, J. Chem. Phys. 61, 4750 (1974). 